\title{
Undulatory fish swimming: from muscles to flow
}

\author{
Ulrike K. Müller \& Johan L. van Leeuwen
}

Experimental Zoology Group, Wageningen Institute of Animal Sciences (WIAS), Wageningen University, Wageningen, The Netherlands

\begin{abstract}
Undulatory swimming is employed by many fish for routine swimming and extended sprints. In this biomechanical review, we address two questions: (i) how the fish's axial muscles power swimming; and (ii) how the fish's body and fins generate thrust. Fish have adapted the morphology of their axial musculature for high power output and efficiency. All but the superficial muscle fibres are arranged along curved trajectories, and the myomeres form nested cones. Two conflicting performance goals shape the fibre trajectories of the axial muscles. Maximum power output requires that all fibres contract uniformly. In a bending fish, uniform contraction in a single myomere can be ensured by curved fibre trajectories. However, uniform strain is only desirable if all muscle fibres have the same contractile properties. The fish needs several muscle-fibre types that generate maximum power at different contraction speeds to ensure effective muscle power generation across a range of swimming speeds. Consequently, these different muscle-fibre types are better served by nonuniform contractions. High power output at a range of swimming speeds requires that muscle fibres with the same contractile properties contract uniformly. The ensuing helical fibre trajectories require cone-shaped myomeres to reduce wasteful internal deformation of the entire muscle when it contracts. It can be shown that the cone-shaped myomeres of fish can be explained by two design criteria: uniform contraction (uniform strain hypothesis) and minimal internal deformation (mechanical stability hypothesis). So far, only the latter hypothesis has found strong support. The contracting muscle causes the fish body to undulate. These body undulations interact with the surrounding water to generate thrust. The resulting flow behind the swimming fish forms vortex rings, whose arrangement reflects the fish's swimming performance. Anguilliform swimmers shed individual vortex rings during steady swimming. Carangiform swimmers shed a connected chain of vortex rings. The currently available sections through the total flow fields are often not an honest representation of the total momentum in the water - the wake of carangiform swimmers shows a net backward momentum without the fish accelerating suggesting that our current picture of the generated flow is incomplete. To accelerate, undulatory swimmers decrease the angle of the vortex rings with the mean path of motion, which is consistent with an increased rate of backward momentum transfer. Carangiform swimmers also enlarge their vortex rings to accelerate and to swim at a higher speed, while eel, which are anguilliform swimmers, shed stronger vortex rings.
\end{abstract}

\author{
Correspondence: \\ Ulrike Müller, \\ Experimental Zoology, \\ Wageningen \\ University, \\ Marijkeweg 40, 6709 \\ PG Wageningen, The \\ Netherlands \\ Tel.: + 00313174833 \\ 35 \\ Fax: + 00313174839 \\ 55 \\ E-mail: ulrike. \\ muller@wur.nl \\ Received 21 Jun 2005 \\ Accepted 16 Feb 2006
}

Keywords: flow visualization, muscle morphology, muscle strain, swimming kinematics, undulatory swimming, wake structure 


\section{Introduction}

What is undulatory swimming? A biomechanical review

How to study the mechanics of undulatory swimming?

The mechanics of swimming: thrust, drag and hydrodynamic efficiency

How does undulatory swimming work: the main questions

How to power swimming: which muscle architecture best suits

Conceptual background

Observation

90

Hypothesis

90

Experiments and models

Conclusions

How to generate thrust: how do water and undulating body interact to propel the fish 93

Test: from flow patterns to propulsive power $\quad 96$

$\begin{array}{ll}\text { Measuring propulsive forces directly } & 97\end{array}$

$\begin{array}{ll}\text { Predicting propulsive forces from theory } & 97\end{array}$

$\begin{array}{ll}\text { Conclusions } & 97\end{array}$

$\begin{array}{ll}\text { Conclusions and the next challenges } & 98\end{array}$

How to power undulatory swimming? $\quad 98$

How to control undulatory swimming? $\quad 99$

How efficient is undulatory swimming? $\quad 99$

$\begin{array}{lr}\text { Acknowledgements } & 100\end{array}$

$\begin{array}{ll}\text { References } & 100\end{array}$

\section{Introduction}

\section{What is undulatory swimming? A biomechanical review}

Many organisms swim by running lateral waves of undulation down their body, making it the most common swimming style amongst fishes. Fish generate a range of body waves to suit their body shape, locomotory requirements and propulsion mechanisms (Fig. 1; for a review see Wardle et al. 1995). Undulatory swimming is commonly used for (fast) routine swimming and extended sprints (for a review see Lindsey 1978; Videler 1993). At low swimming speeds, fish augment with or switch to finned propulsion, either oscillating or undulating their fins (e.g. Drucker and Jensen 1996; Thorson et al. 2004).
An important goal of biomechanical research into undulatory swimming is to understand how muscle contractions lead to a body wave and how the body wave interacts with the water to generate propulsive forces. This process of transforming muscle forces into propulsive forces consists of three steps: (i) the muscles generate forces; (ii) muscle forces are transmitted to the propulsive structures, such as the tail; and (iii) propulsive structures push against the water, transforming motor forces into propulsive forces or thrust. These three steps correspond to the three main components of any propulsion system: motor, transmission and propeller (Wainwright 1983). Each function comes with its own set of design and performance requirements, which can be mutually exclusive. To understand the functional morphology of the fish's swimming apparatus, the biomechanist must disentangle which structures 
(a)
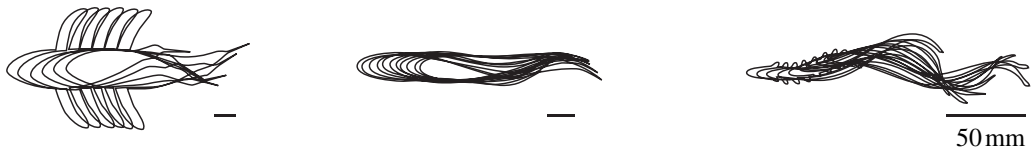

(b)
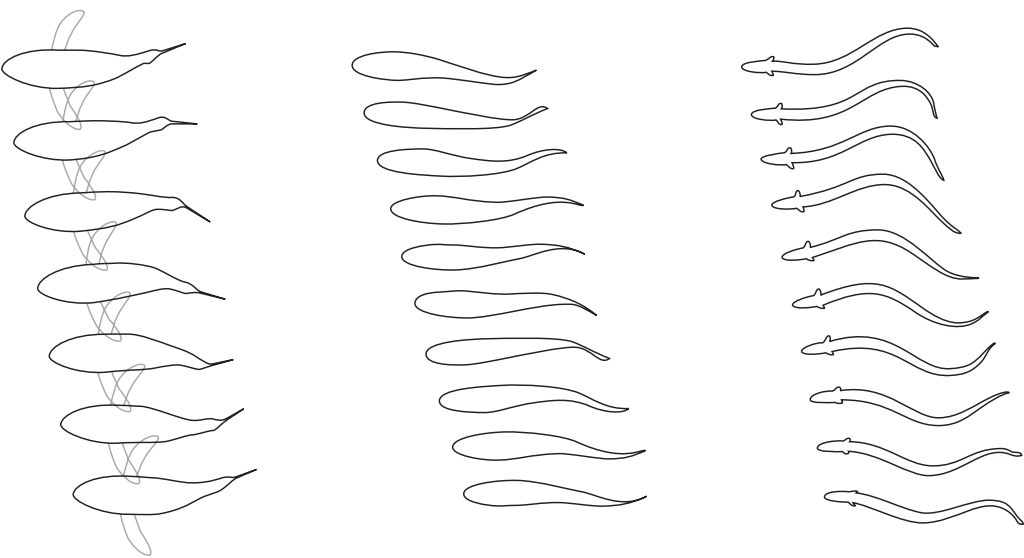

(c)
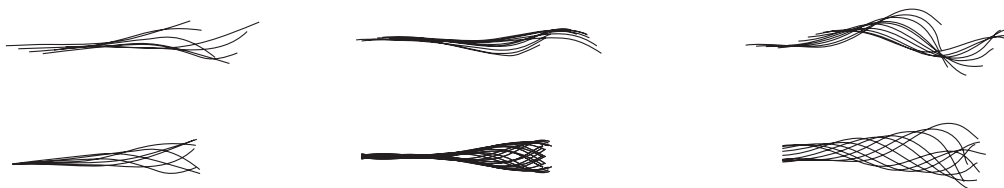

(d)
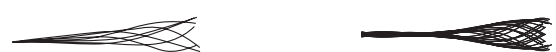

Figure 1 Swimming kinematics for a complete tail beat of three undulatory swimmers. Elongate, slender swimmers generate a short body wave with a wide amplitude envelope along most of the body, e.g. eels and fish larvae (Gray 1933; Müller and van Leeuwen 2004). At the other extreme, fish with high bodies and lunate tails generate a relatively long body wave with a more narrow amplitude envelope (e.g. Dewar and Graham 1994; Donley and Dickson 2000). Left column: yellowfin tuna Thunnus albacares (after Dewar and Graham 1994). Body length $L=0.44 \mathrm{~m}$; swimming speed $U=$ $0.46 \mathrm{~m} \mathrm{~s}^{-1}$; time interval $\Delta t=0.07 \mathrm{~s}$; body wavelength $\lambda=1.24 \mathrm{~L}$; tailbeat amplitude $A=0.18 \mathrm{~L}$; stride length $S=$ 0.4 L. Middle column: saithe Pollachius virens (after Videler and Hess 1984). $L=0.35 \mathrm{~m} ; U=0.63 \mathrm{~m} \mathrm{~s}^{-1} ; \Delta t=0.02 \mathrm{~s}$; $\lambda=1.02 \mathrm{~L} ; A=0.09 \mathrm{~L} ; S=0.8 \mathrm{~L}$. Right column: butterfish Pholis gunnelus (after Gray 1933). $L=0.13 \mathrm{~m} ; U=$ $0.10 \mathrm{~m} \mathrm{~s}^{-1} ; \Delta t=0.05 \mathrm{~s} ; \lambda=0.85 \mathrm{~L} ; A=0.12 \mathrm{~L} ; S=0.4 \mathrm{~L}$. (a) Outlines of dorsal views in an earth-bound frame of reference. The scale bar indicates $50 \mathrm{~mm}$. (b) As in (a), with a vertical shift to show individual outlines. (c) Midlines of the fish outline in an earth-bound frame of reference. (d) Midlines of the fish outline in a fish-bound frame of reference, indicating the amplitude envelope of the body wave.

and processes fulfil which of the three propulsive functions.

Identifying individual propulsive functions is complicated by the highly integrated propulsion systems of most undulatory swimmers (Jordan 1996; Schultz and Webb 2002). In a highly integrated propulsion system, there is a complex spatial and temporal overlap of the main propulsive functions. Several propulsive functions might be fulfilled by the same structure. For example, the axial muscles generate forces (motor function) and then transmit these forces along the myotome (transmission function) to the skin and tail. But the same propulsive function might also be distributed over many structures: force transmission from the motor to the propeller takes place via the myomeres and myosepta, the horizontal septum and other septa, the axial skeleton and the skin (Long 1995; Long et al. 1996, 2002a).

The degree of functional integration is related to how localized or distributed the propeller is along the fish's body. Most of the body contributes to generating thrust in undulatory swimmers that use a wide amplitude envelope (e.g. Lighthill 1960). Consequently, there is considerable spatial overlap and integration of the motor, transmission and propeller functions. Conversely, fish with a narrow amplitude envelope are supposed to generate thrust mainly with their posterior body; in the most extreme case, only the tail acts as the propeller 
(e.g. Yates 1983). Tunas most closely approximate such a spatial separation between motor and propeller: the tail acts as the main propeller, transforming the power generated by the axial muscles into propulsive power. Hence, muscle power, generated by the anterior bulk of muscles, must be transmitted posteriorly to the tail before it can be converted into propulsive power. To this end, the tuna red muscle fibres attach via anterior and posterior oblique tendons to the vertebral column, and the most posterior muscles in tunas coalesce to form the great lateral tendons (Fierstine and Walters 1968; Westneat et al. 1993).

\section{How to study the mechanics of undulatory swimming?}

All propulsive components - the structures that fulfil the functions of motor, transmission and propeller - contribute to the overall swimming performance. Swimming performance is an emergent property of, amongst others, the physical and physiological characteristics of the swimming muscle, soft tissue and skeletal elements and the fluidbody interaction (Jordan 1996). To tease apart how the various components and their interactions determine performance, it is useful to combine experimental techniques with mechanical and mathematical models of undulatory swimmers. This integrated approach allows scientists to manipulate directly and often independently the various factors that contribute to overall swimming performance to study their effect.

In the last two decades, several new techniques have been used to understand how axial muscles power swimming and how this power is transmitted. Electromyographic (EMG) studies have been combined with sonomicrometry (Coughlin et al. 1996) and the workloop technique (Josephson 1985) to measure how the function of axial muscles depends on their position in the body (for a review see Altringham and Ellerby 1999; Coughlin 2002). Detailed anatomical studies (e.g. Westneat et al. 1993; Shadwick et al. 2002; Gemballa and Röder 2004) and mathematical modelling (inverse dynamic models predict forces from movements: Hess and Videler 1984; Cheng et al. 1998; Pedley and Hill 1999; forward dynamic models predict movements from forces: Bowtell and Williams 1991; Jordan 1992, 1996; Long et al. 2002a) have shed further light on force generation and transmission. Kinematic studies have been combined with experi- mental and computational flow analysis to estimate the forces exerted by the body on the water (e.g. Liu et al. 1996, 1997 for tadpoles; Carling et al. 1998 for eel).

This biomechanical review summarizes the current state of the biomechanics of undulatory swimming. It shows in how far we understand the swimming design of fish, and it points out unanswered questions.

\section{The mechanics of swimming: thrust, drag and hydrodynamic efficiency}

Before one can attempt to build a mechanistic model of undulatory swimming, it is useful to define the basic mechanical concepts of swimming. Any swimming organism has to deal with the water's viscosity and density. Water is about 800 times denser than air and 20 times less viscous at $20^{\circ}$. The relative importance of inertial vs. viscous forces is expressed by the Reynolds number, which is defined as:

$$
R e=\rho U L / \mu,
$$

where $\rho$ is the density of the water, $U$ the swimming speed, $L$ the body length of the undulatory swimmer and $\mu$ the (dynamic) viscosity of water. We will focus here on adult fish, which are large and fast enough to swim at $R e$ above 1000 in the so-called inertial flow regime, in which viscous forces are negligible (Fuiman and Batty 1997; McHenry and Lauder 2005). To simplify the physics, we will focus on swimming in a straight line.

When a fish undulates its body, these undulations generate hydrodynamic forces that act on the body. These forces can be decomposed into two components, one along and one perpendicular to the mean path of motion. The component along the path of motion is the sum of thrust and drag, the perpendicular component is the sum of the sideways forces.

The fish can control how much thrust is generated by adjusting the speed with which the body wave travels down the body (Lighthill 1969; Müller et al. 2002). If the wave speed is lower than the swimming speed, the fish generates not thrust but drag and decelerates (Lighthill 1969). If the wave speed exceeds the swimming speed, the fish generates thrust. If total thrust and drag along the body balance each other over the duration of one complete tail beat, then the fish swims steadily: it maintains a constant mean speed over successive tail beat cycles. If the generated thrust exceeds drag, the fish accelerates. 
Due to the cyclic nature of the body undulations, hydrodynamic forces oscillate over a complete tail beat cycle (Lighthill 1960; Videler and Hess 1984). In other words, during steady swimming, instantaneous thrust and drag and their distribution along the body are not constant. The oscillation of instantaneous thrust and drag in time and space implies that the role of a given body segment might change during the tail beat cycle from 'propeller' that generates thrust to 'brake' that generates drag' and vice versa. Consequently, in all undulatory swimmers, except the extreme cases such as thunniform swimmers, it makes little sense trying to distinguish between propeller, which generates thrust, and fuselage, which generates drag (Schultz and Webb 2002).

Not only thrust and drag, but also instantaneous sideway forces vary in time and along the body. These oscillating sideway forces can result in an instantaneous net torque and net force on the body that cause the fish to yaw and heave (Fig. 1). If the sideway forces to the left and right cancel out over the duration of one tail beat cycle, the fish swims on a straight mean path of motion.

When a fish starts to swim, it needs to accelerate its mass until the fish reaches the desired steady swimming velocity. Thus, the fish is gaining forward momentum equivalent to its mass times its forward velocity. The momentum balance requires that there is an equal and opposite momentum shed in the water. Once the fish swims steadily at a constant mean velocity over the swimming cycle, the fish would move forward indefinitely due to forward momentum it gained during the acceleration phase if it were not for the drag forces exerted by the water. To compensate for the momentum loss due to drag in order to maintain a constant velocity, the fish must continue to generate forward momentum. The rate at which the fish generates forward momentum is equivalent to the generated thrust (force $=$ rate of momentum). If the fish's thrust compensates exactly for the drag acting on its body, then there is no net change in the fish's momentum and therefore, the water behind a fish swimming at a constant velocity should contain no net forward or back momentum.

An imbalance between thrust and drag forces causes the fish to change velocity. In other words, if a net force, e.g. drag, is exerted on the fish, then the fish does not generate forward momentum at a high enough rate to compensate for the drag forces acting on its body. The fish decelerates, and this loss of forward momentum will manifest itself in the water behind the decelerating fish as a net flow in the direction of motion of the fish. These considerations on how changes in the fish's momentum translate into changes in the water's momentum will become important again later in this review on how to interpret cross-sections through the flow visualized around swimming fish.

The power generated by the fish to swim is the product of the thrust force and the forward velocity of the fish (power $=$ force times velocity). This power is lower than total hydrodynamic power because the fish generates also backward and sideway momentum in the water, which contributes to the total power transferred by the fish to the water. The ratio of this propulsive power to the sum of all hydrodynamic power can be defined as hydrodynamic (or Froude) efficiency (Lighthill 1969). Hydrodynamic efficiency is also affected by the force component perpendicular to the mean path of motion. This sideways component induces yaw and heave, which can lead to weak thrust forces acting on the snout (Long et al. 2002b). However, large sideway forces lower rather than increase hydrodynamic efficiency.

Thus, the muscle must deliver more power than the propulsive power that is ultimately generated by the propeller. The difference between muscle and propulsive power is increased further by losses that occur in the force-transmitting structures because the deformations that accompany force transmission are not perfectly elastic and therefore not entirely loss-free. Further losses are incurred because the muscles convert the energy of ATP hydrolysis not only into mechanical work but also into heat. All these losses need be taken into account to determine the total swimming efficiency.

Low costs of transport can be achieved by streamlining the body and by swimming movements that minimize drag. To further reduce costs of transport, the rate of energy spent on generating thrust can be reduced by increasing the mass of water that is accelerated backward.

\section{How does undulatory swimming work: the main questions}

In order to swim, fish need a motor - the axial muscles - that generate forces to power the propeller - the body and tail fin. Most fish possess a narrow strip of red muscle fibres (aerobic, slow twitch) that power continuous swimming; the bulk 
of the axial musculature is made up of white muscle fibres (anaerobic, fast twitch) that power fast starts and sprints. For a review on the contractile properties of these two muscle-fibre types and on how their contractile behaviour matches the functional requirements of undulating a fish body, the reader is referred to a review by Coughlin (2002). This review will focus instead on muscle architecture: how should the fish arrange its muscle fibres to build an efficient motor that undulates its body.

Fish need a motor not only with enough power, but also with the correct gearing to transmit power to their propeller. Gearing codetermines the load experienced by the activated muscle fibres and hence their contraction speed and power. Muscle fibres generate maximum power within a fibrespecific range of contraction speeds. This contraction speed codetermines the frequency of the body wave and therefore the swimming speed. Wakeling and Johnston (1999) provide an overview of gearing in fish swimming. Brainerd and Azizi (2005) provide an accessible introduction into how fibre architecture affects gearing in their study on a non-teleost undulatory swimmer, the aquatic salamander Siren lacertian.

Fish need to transmit muscle forces to the propeller. If propeller and motor are spatially separated, force transmission pathways are more readily identifiable because fewer propulsive functions are integrated in one structure. Tuna and lamnid sharks lend themselves for morphological studies to identify which structures transmit muscle forces (Westneat et al. 1993; Shadwick et al. 2002; Donley et al. 2004). Detailed anatomical studies also exist for other fish species, providing the structural basis for the possible pathways of force transmission (e.g. Gemballa and Röder 2004). Further indications about the distribution of muscle forces and power have come from the combination of EMG measurements with kinematic and sonomicrometry studies (Ellerby and Altringham 2001, for a review see Altringham and Ellerby 1999) and inverse dynamic models of fish swimming (Hess and Videler 1984; Cheng et al. 1998; for a review see Pedley and Hill 1999). However, there is still much debate around those non-thunniform swimmers with considerable spatial overlap between propeller and motor. The debate centres around whether or not the posterior axial muscles act as motor or as force transmitter (Rome et al. 1993 vs. van Leeuwen et al. 1990). This controversy has been covered in reviews by van Leeuwen (1995), Altringham and Ellerby (1999) and Coughlin (2002).

After being transmitted to the propeller, the muscle forces need to be converted into thrust by the propulsive structures of the fish, which in undulatory swimmers are the body and the unpaired fins. This review will present the current knowledge of how fish generate flow patterns and how these flow patterns relate to swimming performance.

\section{How to power swimming: which muscle architecture best suits the task of bending the body?}

\section{Conceptual background}

A long-standing problem in fish swimming has been how to explain the unique morphology of the fish's axial musculature in terms of its function during undulatory swimming. We will focus on two architectural features: the unique shape of teleost myomeres and the curved trajectories of the muscle fibres within the myotome. Concerning the fibre trajectories, there are two conflicting design demands: excellent acceleration performance or good swimming performance across a range of speeds. On one hand, fish might benefit from good acceleration performance to power escape responses or feeding strikes. High acceleration requires high muscle power. For maximum muscle power, the fish should recruit all its unilateral muscle fibres equally. Equal recruitment implies uniform strain along the muscle-fibre trajectories. Muscle fibres should not do work by forcefully stretching other muscle fibres. Such internal deformations of the myomeres waste muscle energy that otherwise would be available to power swimming.

On the other hand, fish need to be able to swim effectively at a range of speeds. Swimming speed scales with tail beat frequency and tail beat amplitude: an undulatory swimmer achieves higher speeds by mainly increasing its tail beat frequency, and to a lesser extent its tail beat amplitude [eel (Anguilla rostrata, Anguillidae): Tytell 2004a; tuna (Euthynnus affinis, Thunnidae): Donley and Dickson 2000]. To achieve a higher tail beat frequency, the muscle needs to contract faster to contract by the same length. To achieve a higher tail beat amplitude, the muscle needs to contract over a larger length in the same time. Either way, higher swimming speeds lead to higher strain rates in the muscle 
fibres. Strain rates at the maximum swimming velocity should match the contraction speeds for maximum power output of the muscles fibres. In order to operate their swimming muscles near their optimum contraction speed for high power output, fish need to distribute the orientation and contractile properties of their muscle fibres so that there is always a certain proportion of fibres that operates optimally (with respect to efficiency or power output) at the contraction rate required to maintain a given swimming speed. Hence, high swimming proficiency over a range of swimming speeds requires a range of muscle-fibre types and fibre orientations, as well as non-uniform strain distributions within the myotome.

\section{Observation}

In most teleosts, the myomeres are folded in the form of nested triple cones. A triple cone consists of one anterior cone, flanked on the top and bottom by two posterior cones (Fig. 2). The cones become progressively more pointed towards the tail. Myomeres closer to the tail have deeper posterior cones, and the anterior cone often changes from having a single apex, which touches the vertebral column, to becoming bifid, with both apices lying free in the musculature (van Leeuwen 1999). In many fish, the red muscle forms a superficial narrow strip, and only white muscle fibres are generally present near the apices of the cones, with a few notable exceptions such as tunas (Graham et al. 1983).

Fish possess at least two muscle-fibre types - red and white, and generally, several intermediate types (e.g. Johnston et al. 1977; Akster 1985). While superficial muscle fibres run roughly parallel with the body axis, the bulk of the muscle fibres, and especially the fibres in the deeper portions of the myomere make variables angles up to a maximum of $40^{\circ}$ (van der Stelt 1968; Alexander 1969). This fibre angle depends on the position of the muscle fibre in the body, and Alexander (1969) described the fibres of successive myomeres as forming curved trajectories that run through the body of teleost fish and sharks.

\section{Hypothesis}

Alexander (1969) and van der Stelt (1968) formulated the uniform strain hypothesis to explain this complex fibre arrangement: uniform strain enables all fibres of the same type to contribute equally to generating power and ensures that the fibres do not work against each other. If the fish bent like a

(a)

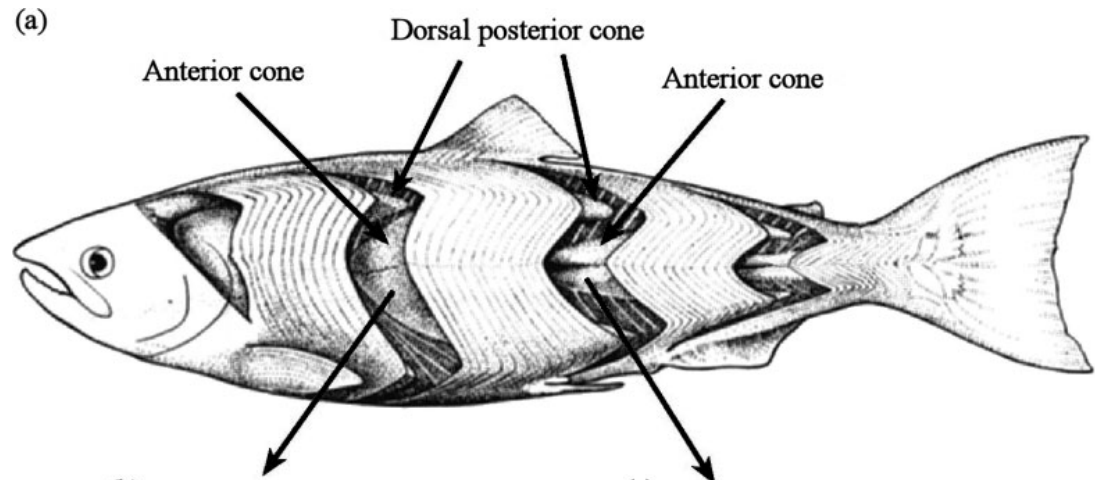

(b)

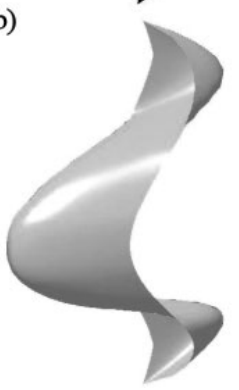

(c)

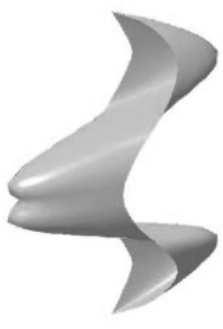

Figure 2 (a) White myomeres of king salmon, adapted from Greene and Greene (1913). (b) Simulated shape of a mechanically stable anterior myoseptum. (c) Simulated shape of a mechanically stable posterior myoseptum. (Figure adapted from van Leeuwen 1999.) 
homogenous beam, then the beam would be compressed on the concave inside of the bent and stretched on the convex outside of the bent, while the neutral axis in the middle of the beam maintains its original length (see Alexander 1969 for a more detailed explanation of beam theory). In a bending fish, if the white muscle fibres were arranged parallel to the body axis, fibres close to the axis would experience lower strains than the more superficial fibres, with the fibres adjacent to the body axis unable to contribute useful work because they experience essentially no strain. To contribute equally, deeper muscle fibres must have different orientations from more superficial fibres. Hence, the requirement of uniform strain leads to curved muscle-fibre trajectories.

These curved fibre trajectories affect the shape of the myomeres. If the myomeres were arranged simply in stacks of flat slabs, then the differences in muscle-fibre direction, activation and cross-sectional area would lead to different forces exerted by the muscle fibres on either side of the myoseptum (van Leeuwen 1999). This difference in forces would then lead to considerable deformations of the myoseptum when the muscle fibres contract. The exact internal deformation of myosepta in a swimming fish depends also on the complex loading by external and internal forces and the mechanical properties of the various tissues, which are in part controlled by the nervous system. To avoid wasteful internal deformation and thereby to increase the muscle power available for useful propulsive work, myoseptum shape and therefore myomere shape and stiffness need to be adjusted to suit the aforementioned force differences across the myoseptum. Each myomere needs to assume a resting shape that minimizes internal deformation when the muscle fibres contract. This demand is summarized in the mechanical stability hypothesis (van Leeuwen and Spoor 1992, 1993; van Leeuwen 1999): myomere contraction should affect overall myomere shape as little as possible, i.e. shape must be mechanically stable. The mechanical stability hypothesis predicts that the nested triple-cone shape of the fish's myomeres is a result of the distribution of muscle-fibre orientations and differences in the force production across the myoseptum.

\section{Experiments and models}

The uniform strain hypothesis can in principle be tested by measuring longitudinal strain in the muscle fibres of the fish (for a review see Coughlin 2002). However, these experiments are so far inconclusive. While one study confirmed uniform strains in carp (Cyprinus carpio, Cyprinidae) (Rome and Sosnicki 1991), another study concluded that carp white muscles behaved like a bending beam strain was not constant but proportional to the distance from the median plane (Wakeling and Johnston 1999). Rome and Sosnicki (1991) bent freshly killed fish into postures assumed during undulatory swimming and quantified muscle strains microscopically once rigor mortis had set in. This approach assumes that in rigour muscle strains due to body curvature honestly represent in vivo strains in an active muscle during swimming. Wakeling and Johnston (1999) used sonomicrometry to quantify strain, a method that provides spot checks, usually at two to four locations in the more superficial white muscle. Other sonomicrometry studies also concluded that fish bent like a beam with uniform material properties (for a review see Goldbogen et al. 2005). However, such a beam-like bending behaviour during high-power manoeuvres would lead to minute strains in fast muscle fibres near the medial plane, severely limiting these fibres' capacity to power undulatory swimming.

The accuracy of sonomicrometry measurements depends on the exact alignment of the sonomicrometry crystals along the muscle-fibre trajectories and on exact position of the crystals relative to the adjacent tissue during the strain cycle. One sonomicrometry study published so far measured strain along the actual local muscle-fibre trajectories of fish, achieving a $\leq 10 \%$ accurate alignment of the sonomicrometry crystals with the muscle-fibre directions (Ellerby and Altringham 2001). This lack of alignment might partially explain why Wakeling and Johnston (1999) occasionally found considerable deviations between measured strains and the strain predicted by beam theory for the deeper white muscle fibres. The most reliable strain records are available for superficial fibres, which have fibre trajectories that are less inclined relative to the body axis than deep fibres. The arrangement of the slow red muscle fibres parallel to the body axis results in a strain behaviour very much like a bending beam (for a review see Coughlin 2002). To date, we still have no satisfactory measurements of the threedimensional strain field in the swimming muscles that might serve as direct evidence for the uniform strain hypothesis. 
Indirect qualitative evidence for the uniform strain hypothesis comes from computations by van der Stelt (1968). van der Stelt quantitatively predicted the directions of muscle fibres and myosepta assuming that: (i) the all fibres would have the same longitudinal strain; and (ii) that the myosepta are inextensible yet pliable. His two-dimensional computations were limited to very small body curvatures, yet they show a remarkable similarity with horizontal sections through the axial muscles of lamprey and some teleost species. This qualitative agreement of prediction and actual architecture can be considered as limited evidence in favour of the uniform strain hypothesis.

The most-frequently cited paper concerning the uniform strain hypothesis is Alexander (1969). Alexander compared the average strains of three differently shaped muscle-fibre trajectories, computed for large body curvatures, but for simplified internal deformations of the body. However, by calculating average strain along the entire trajectory, Alexander allowed local strain to vary along the trajectory, although he did not determine by how much strain might vary. This variable strain along a trajectory means that Alexander's predictions of similar strains in different trajectories do not provide strong support for the uniform strain hypothesis, leaving the similarity between van der Stelt's predictions and actual muscle morphology as the only, although limited, evidence so far in favour of the uniform strain. Alexander (1969) also calculated that superficial red muscles experience on average a four times higher strain than the white muscles at the same location along the body. This gearing ratio of 1:4 arises from the combined effect of fibre orientation and the position of a muscle fibre relative to the body axis. This value differs from the measurements of Wakeling and Johnston (1999), who found a gearing ratio of 1:2 in carp.

The two approaches of van der Stelt (1968) and Alexander (1969) are fundamentally different. van der Stelt predicted form from functional requirements; predicted morphology was then tested against observed morphology. Alexander predicted function from form, in his case a simplified representation of the observed muscle architecture. Alexander's predicted average strains along the observed trajectories then needed to be tested against measured strains.

Van Leeuwen (1999) produced a forward dynamic model that predicts the myomere shape from the mechanical stability hypothesis. This hypothesis proposes that the nested triple-cone shape of fish myomeres is, among other factors, a consequence of the helical fibre arrangement. The muscle-fibre arrangement is taken as a given input parameter. Thus, myomere shape is predicted from this fibre arrangement, rather than predicting fibre trajectories, which would be required to test the uniform strain hypothesis. The model calculates the loads on a myoseptum that is situated between two contracting myomeres based on a fair approximation of the fibre trajectories measured in fish. The model makes several assumptions about this loading pattern: (i) the anterior muscle fibres in the region of the anterior cone exert a slightly higher force on the myoseptum than their posterior neighbours because they were activated earlier (based on EMG observations) and because they have a greater cross-section; (ii) the more posterior muscle fibres in the regions of the posterior cones exert higher forces than their neighbouring anterior fibres (attributed to a greater pre-stretch effect that enhances force output with virtually no difference in timing); and (iii) external forces from the surrounding water are neglected (but skin stiffness is included). The initial condition of the model is a block of flat myomeres, separated by a myoseptum. The model computes the shape of the myoseptum that is in mechanical equilibrium with the muscle-fibre loads.

The model of van Leeuwen (1999) reaches good agreement with the actual shapes of the myomeres in the salmon and yields qualitatively correct predictions of the myotome (Fig. 2), providing support for the mechanical stability hypothesis. The model predicts the triple-cone shape that is typical for bony fish, the changes in cone shape from a prominent anterior cone in myomeres near the head to more prominent posterior cones in myomeres closer to the tail, an anterior cone with a single apex near the head and with two apices in myomeres nearer the tail (van Leeuwen 1999). The mechanical stability hypothesis (van Leeuwen and Spoor 1992, 1993) can explain how curved musclefibre trajectories lead to the observed triple-cone myomere shape. Teleost fish might possess coneshaped myomeres because such a resting shape minimizes wasteful internal mechanical deformation during contraction.

\section{Conclusions}

The currently available studies are not sufficient to test the uniform strain hypothesis. To show that the 
helical fibre trajectories in the axial musculature ensure a fairly uniform strain in muscle-fibre groups of the same type within each myomere, a quantitative 3-D model is required that calculates local strains for large deformations and shows the consequences of the curved fibre paths for the work output and strain variations in the fast muscle mass.

With the exception of tunas and lamnid sharks (Donley et al. 2004), the prediction of curved fibre trajectories only applies to white, but not to red muscle fibres, which run almost parallel to the body axis. Red muscle fibres are far away from the body axis and form a thin strip, which results in fairly uniform strains across the thickness of the red muscle strip. Hence, red muscle fibres do not need complex fibre trajectories to keep strain uniform. The posterior taper of the fish's body decreases the distance between red muscle and body axis, which should lead to lower strain in the posterior red fibres compared with the more anterior fibres. However, the effect of the tapering body on local muscle strain is almost exactly compensated for by the posteriorly increasing body wave amplitude. The posterior increase in body wave amplitude raises body curvature, which in turn raises strain (e.g. van Leeuwen et al. 1990; Katz et al. 1999). This match between body taper and increased body wave amplitude ensures fairly uniform strain not only across, but also along the red muscle strip.

Uniform strain is only desirable if the fish were to use all muscle fibres under identical contraction conditions at the same time. However, there is an alternative design criterion for the muscle architecture, the aforementioned gearing. If strain were completely uniform across the entire myotome, then the fish would have only one gear. Alternatively, fish might arrange a proportion of their muscle fibres in a manner that creates a range of strains for a given body curvature. Most teleosts have at least two muscle-fibre types, each with their own fibre arrangement, to suit the fish's range of swimming speeds and tail beat frequencies (Johnston 1991). These two fibre types provide carp with a gear ratio of 1:2 (Wakeling and Johnston 1999). Having a range of gear ratios for a range of swimming speeds and having uniform strain to maximize power output are mutually exclusive designs. Fish probably choose a middle path, which might explain the aforementioned discrepancy between uniform strain hypothesis and observed variation in strain.

Although uniform strain vs. gearing has not yet been established as the main design principle that governs muscle-fibre trajectories, we now have a better understanding of why fish myomeres are arranged in nested triple cones. The model of van Leeuwen shows that this design can be explained by the stability hypothesis - the myomeres adopt a shape that avoids large internal deformation of the myomere and that keeps myomere shape stable during contraction. The model receives additional credibility because it also predicts several peculiarities of teleosts and sharks that were used neither to formulate the original mechanical stability hypothesis nor to design the model. A weak skin leads to concavities in the body when the axial muscles contract (van Leeuwen 1999). These concavities are detrimental to the streamlining of the body. Concavities can be avoided in three ways, a combination of which can be found in all fishes. First, the fish can develop bones to stiffen the body against the compressive load of the muscles. Second, in the absence of bones, the skin must become extra stiff. Third, fish with a round body cross-section are less susceptible to these concave grooves along their midline than a high and flat fish. Sharks, which cannot form intermuscular bones, show option two and three: they have a very stiff skin compared with most teleosts (Wainwright et al. 1978), and sharks usually have a round body cross-section. Lamnid sharks do not adopt the elliptic streamlined body cross-sections typical for tunas and other highperformance cruisers (Donley et al. 2004) despite an otherwise almost perfect convergence between tunas and lamnid sharks. Many teleosts adopt option one: they form bones to stiffen those sections of the myosepta and horizontal septum that are loaded in compression (van Leeuwen 1999).

In conclusion, we now have strong indications that the mechanical principle underlying the peculiar myotome architecture of fish is mechanical stability. This understanding also led to the insight that myotome shape, the presence of bones inside the myomeres and the cross-sectional shape of fish are all related to stabilizing the shape of the myomeres during contraction.

\section{How to generate thrust: how do water and undulating body interact to propel the fish}

\section{Conceptual background}

Flow visualization has become an important tool to study swimming. However, the interpretation of wake shape requires some hydrodynamic back- 
ground knowledge. Any object moving through water will shed a wake. In the most simple case the fish coasts without moving its body - the fish's boundary layer will slip off at the fish's trailing edge and form a wake. A coasting fish experiences drag forces, which decelerates the fish. The forces exerted by the coasting fish on the water add forward momentum to the water, and behind the fish forms a drag wake. If the fish undulates its body, then this motion will cause additional water movements, which will alter the amount of momentum transferred to the water and which will thereby affect the wake.

The wake indicates swimming performance. Wake structure depends on the net momentum transferred to the water: the momentum gained by the fish must balance the momentum shed in the water (Gopalkrishnan et al. 1994). During swimming at a constant average velocity, the time- and space-averaged momentum shed into the wake is zero over one tail beat cycle. An accelerating fish will shed a wake with a net backward momentum, a decelerating fish a wake with net forward momentum. Fish that have a higher density than water generate a downward momentum in the wake to avoid sinking (Wilga and Lauder 2002).

Momentum transfer occurs at more than one location: fish use their body and their fins in various combinations for different swimming behaviours. All these structures generate drag or thrust or alternate between drag and thrust production over the swimming cycle. Hence, they all shed a wake (for a review see Lauder and Drucker 2002). These wakes interact and combine to form the total wake behind the fish (e.g. Drucker and Lauder 2001).

The most striking structures in a wake are vortices, which are the result of the fish's momentum transfer to water. The pattern in which these vortices arrange depends not only on the amount and direction of the transferred momentum but also on when and where this momentum is shed into the wake. Vortices can be recognized in a wake by the locally elevated vorticity. Vorticity is a measure for the rotational velocity of the flow, and it is high in the core of a vortex.

Vortices cannot end freely in the flow and therefore need to either remain connected to the fish or form vortex rings (Helmholtz condition, Prandtl and Tietjens 1934). A vortex ring is characterized by its ring diameter, the diameter of its core and by its strength. Vortex strength is equivalent to the integral of vorticity over the surface area of the vortex core (for further details on vortex strength and energy see Lamb 1932; Spedding et al. 1984; Müller et al. 1997). Vortex rings form around or induce a jet flow through the centre of the ring. This jet is often oriented at an angle with the mean path of motion of the fish, the so-called momentum angle. Vortex rings in a wake can remain separated from each other or form interconnected chains (Fig. 3).

\section{Observation}

Two distinct wake patterns have been observed behind undulatory swimmers (Fig. 3). When swimming steadily, eels shed two separate vortex rings per tail beat cycle (Müller et al. 2001: Anguilla anguilla, Anguillidae; Tytell 2004a; Tytell and Lauder 2004: Anguilla rostrata, Anguillidae). Once shed, these rings move away from the mean path of motion. Trout (Oncorhynchus mykiss, Salmonidae), mullet (Chelon labrosus, Mugilidae) and mackerel (Scomber japonicus, Scombridae) shed a chain of connected vortex rings, one chain link per half tail beat (Blickhan et al. 1992; Müller et al. 1997; Nauen and Lauder 2002).

(a)
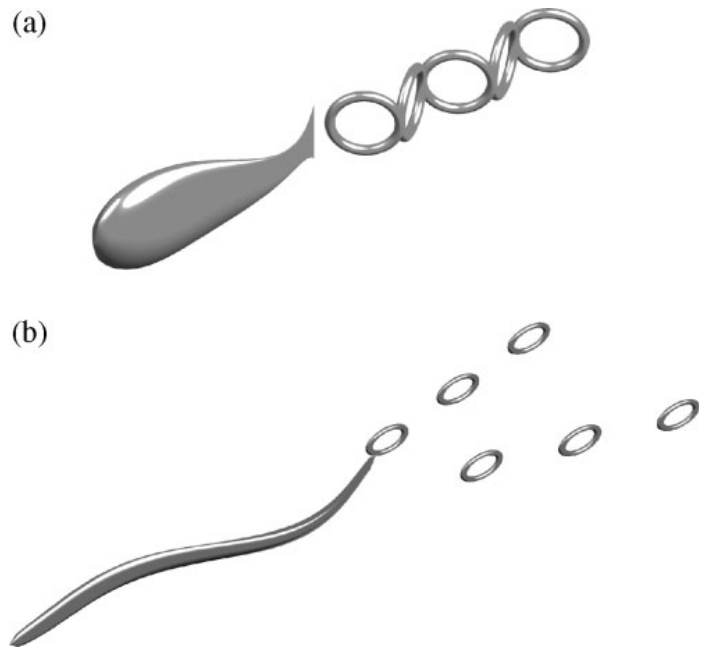

Figure 3 Dorsolateral view at the wakes of undulatory swimmers generated during steady swimming.

(a) Carangiform swimmers shed a chain of connected counter-rotating vortex rings. (b) Anguilliform swimmers shed a wake of individual vortex rings perpendicular to the path of motion. (Image based on flow visualizations by Müller et al. 2001 and Tytell 2004a.) 
During steady swimming, the basic vortex shedding pattern remains the same across a range of swimming speeds (Nauen and Lauder 2002; Tytell 2004b). When eel swims faster, the flow through the vortex rings is faster and more sideway momentum is imparted to the water. However, when estimating the cost per unit distance swum of producing the wake from the flow, Tytell (2004a) finds that this cost seems to increase more slowly with swimming speed than if cost were proportional to the square of swimming speed (kinetic energy of the fish is proportional to its mass times the square of its forward velocity), suggesting that the swimming efficacy increases with swimming speeds. Mackerel double swimming speed not only by almost doubling the strength, but also by increasing the size of their vortex rings and by reducing the rings' momentum angle from $34^{\circ}$ to $27^{\circ}$ (Nauen and Lauder 2002). Increasing the amount of water to which momentum is transferred by increasing vortex ring diameter is more efficient than increasing flow speed or vortex strength: the kinetic energy required to impart a given momentum (=mass times velocity) scales with mass ${ }^{1}$, but with velocity ${ }^{2}$.

During linear accelerations and decelerations, the net momentum in the wake along the mean direction of motion changes from zero to negative (opposite to the fish's direction of motion) and positive (with the direction of motion), respectively, to maintain the momentum balance. Linearly accelerating eels generate a wake that consists of two vortex rings per tail beat cycle (Tytell 2004b), just as during steady swimming. The net backward momentum in the wake manifests itself in the angle between ring axis and mean path of motion: during acceleration, the eel sheds vortex rings that are oriented slightly backward at momentum angles of less than $90^{\circ}$, while during steady swimming the vortex ring axis is oriented perpendicular to the mean path of motion (Tytell 2004b).

Carangiform swimmers decelerate by increasing the momentum angle of their vortex rings (Müller et al. 2002). As the momentum angle approaches $90^{\circ}$, the horizontal cross-sections through the far wake of a mullet resemble the flow pattern behind a steadily swimming eel (Müller et al. 2001).

\section{Hypothesis}

Mechanical and mathematical models of oscillating plates have shown that there are two distinct vortex patterns in the wake that correlate with two performance optima, maximizing thrust and maximizing efficiency (Triantafyllou et al. 1991; Gopalkrishnan et al. 1994; Streitlien et al. 1996). These vortex patterns require a particular motion pattern of the heaving and pitching plate. Wake shape depends on the oscillating frequency $f$, amplitude $A$ and forward speed $U$ of the plate's trailing edge. These quantities are reflected in the Strouhal number (e.g. Triantafyllou et al. 1991):

$$
S t=2 f A / U
$$

The vortex pattern in the wake reflects the propeller's shape and motion pattern of the its trailing edge. So, if the fish wants to maximize thrust or efficiency, it should find a combination of body shape and swimming kinematics that enables it to shed a wake that is optimized for their performance goal. In this case, wake shape should be very similar across the range of undulatory swimmers despite differences in body shape and body wave characteristics as long as these fish have the same performance goal, i.e. either high thrust or high efficiency. If, for example, maximizing efficiency were a general trend in steady swimming, then fish should tune their body shape and swimming kinematics to converge onto the double vortex ring wake. Vorticity is shed at trailing edges of the fish, i.e. its fins and at contraction zones of the body crosssection (e.g. Nauen and Lauder 2001). It then rolls up into vortices, which arrange themselves in well-described patterns (e.g. Triantafyllou et al. 1993).

The predicted optimum wakes apply only to steady swimming. If the fish were accelerating or decelerating, the wake would change because of the different net momentum in the water (Müller et al. 2002; Tytell 2004b). The altered momentum balance will at least affect the net flow velocities in the wake. Model experiments predict that the effects are more profound: by analogy to the wake behind flapping foils (Triantafyllou et al. 1991), small changes in tail beat amplitude, frequency and swimming speed can change wake shape. Furthermore, flow visualization experiments around fish suggest that such changes in wake shape might be brought about by small changes in the body wave when changes in amount and direction of momentum cause vortex rings that are shed in a similar pattern as during steady swimming, to become unstable and form into a qualitatively different wake pattern (Müller et al. 2001; Tytell 2004b). 


\section{Test: from flow patterns to propulsive power}

In order to test this optimum wake hypothesis, it is important to determine propulsive forces and propulsive power and to relate these to body wave kinematics and body shape. Propulsive forces and power can be obtained from the flow patterns around the fish. Since the 1990s, quantitative flow visualization has become an important tool to study fish swimming (e.g. Müller et al. 1997; Drucker and Lauder 1999; Wolfgang et al. 1999). Digital particle image velocimetry (DPIV) provides a valuable glimpse at the swimming performance of a fish and the processes of wake generation. However, this technique has at least two important limitations. First, most records of flow fields show only the flow in one plane, neglecting any flow components through this plane and outside this plane. Second, flow visualization initially only yields information on flow velocity, not forces, and hence is only the first step towards determining propulsive power.

The first limitation became obvious when flow was visualized in a horizontal plane behind steadily swimming mackerel (Nauen and Lauder 2002), trout (Blickhan et al. 1992) and sharks (Wilga and Lauder 2002). These experiments were conducted in a flow tunnel, with the fish swimming against a constant current. Studies in flow tunnels provide scientists with an easy check that the fish do not change swimming speed over the course of several tail beats. Yet, two-dimensional cross-sections through the wake behind these steadily swimming fish clearly contain net backward momentum, creating the misleading impression of a fish that is accelerating and hence generating net backward momentum in the water. However, similar experiments with eel swimming steadily in a flow tunnel yielded cross-sections of the wake that contain no net sideway or backward momentum and therefore give an honest impression of the propulsive momentum balance. We must conclude that the vortex chain pattern behind a steadily swimming carangiform swimmer cannot be the complete wake. The flow patterns observed in fish during carangiform swimming at a constant speed and while decelerating all contain a momentum imbalance: there must be areas containing forward momentum that are not visible in the currently available views of the flow field (e.g. Müller et al. 1997, 2002). Hence, medial cross-sections through the total flow field cannot be considered a reliable indicator of net propulsive momentum, and the complete threedimensional wake structure needs to be mapped to estimate the net momentum transferred by the fish to the surrounding water. The required experimental techniques are now becoming available to record three-dimensional flow (e.g. Pereira et al. 2000; Malkiel et al. 2003).

The second limitation is even more severe. Flow patterns need to be translated into force distributions in order to establish propulsive mechanisms and the location of the propeller, both of which can vary over the duration of a tail beat cycle. In the fish literature, flow analysis so far focused on the vortex pattern in the wake to determine propulsive power (Müller et al. 1997; Drucker and Lauder 1999; Tytell 2004a,b). However, the wake is the result of momentum transfer. If viscous losses and vortex break-up are negligible, then the power contained in the vortices is a fair representation of the amount of propulsive power and even efficiency. However, the wake contains at best indirect information about how and where the momentum was originally transferred to the water, and therefore is less useful when one tries to identify propulsive mechanisms and structures.

Noca et al. (1997) developed a formalism to extract force information from two-dimensional DPIV velocity fields using the flow velocity information around the animal rather than the shape and strength of vortices in the wake. This method should at least be able to deliver a time course of the force generation during the tail beat cycle. Noca's approach has been applied to the flow patterns of a hovering robotic insect, for which net propulsive forces could be measured at the same time (Birch et al. 2004): However, this approach under-predicted the forces developed by a flapping foil by 20$30 \%$ (Birch et al. 2004). Extrapolating forces from a two-dimensional slice through the flow can lead to errors for at least two reasons. First, as in fish, the two-dimensional flow field might not be an honest representation of the total momentum balance. Second, DPIV algorithms estimate flow velocity using statistical methods: they calculate mean flow speeds for small areas in the flow field. DPIV is therefore sensitive to under-estimating flow velocity if there are steep local velocity peaks. Extrapolating two-dimensional DPIV flow information to threedimensional forces may yield good order-of-magnitude estimates, but is not accurate and precise enough to predict force distributions along moving animals. So far, we still have no reliable quantitative 
information about the temporal and spatial distribution of fluid forces along the fish's body to understand how fish swim.

\section{Measuring propulsive forces directly}

There have been few attempts to measure forces directly in undulatory swimmers. Webber et al. (2001) measured differential pressures at the caudal peduncle of a swimming cod (Gadus morhua). McHenry et al. (2003) measured net propulsive force during tethered swimming of an ascidian larva (a chordate with a muscular tail, but not a teleost). However, tethering leads to force distributions on the body that are not representative for free swimming, obliterating any possible correlation between wake shape and swimming performance that is necessary to test the optimum wake shape hypothesis. Furthermore, to understand the nature of the fish's propeller, we need to know the distribution of propulsive forces along the entire body during 'free' swimming, not only the temporal pattern of propulsive force development or the forces at one point.

\section{Predicting propulsive forces from theory}

Without a feasible way to measure the distribution of forces and moments directly, these values can be estimated from fluid dynamic theory. Several successful analytical and numerical models have been developed to estimate fluid dynamic forces from the swimming movements and body shape of the fish. The earliest of these models were analytical and therefore rather limited: they neglected viscosity, did not account accurately for body shape and were only valid for a limited array of swimming movements (Lighthill 1960; Wu 1961). With the steep increase in computer power, numerical solutions have become feasible. A less computationally demanding approach, the vortex lattice method, has been pioneered by Schultz (Schultz et al. 1991; Schultz and Webb 2002), Cheng (Cheng et al. 1998) and Wolfgang (Wolfgang et al. 1999), and has been applied repeatedly to fish swimming. The vortex lattice or panel methods neglect viscosity and hence does not correctly account for the boundary layer structure and associated skin friction around the fish. This review will therefore focus on the most exact approach, numerical solution of the NavierStokes equation. Recently, computational fluid dynamics (CFD) models have come within reach of the biofluid dynamics community (Liu et al. 1996, 1997; Carling et al. 1998). However, several limitations still dog CFD models. First, exact solutions are only possible for laminar flow, which occurs around small or slow fish, up to the size of a few centimetres and swimming at speeds up to a few centimetres per second (Liu et al. 1996, 1997). For larger and faster fish that swim in the turbulent regime, turbulence models need to be implemented in the CFD code to keep the computational demands within realistic limits. But turbulence models lower the accuracy of force estimates. Second, CFD is a numerical approach: it divides the space around the fish up into small parcels and then calculates the flow for each small parcel. This gridding of the three-dimensional space around the fish remains difficult because of the complex and time-dependent movements that need to be mapped, such as the fish's large-amplitude movements during a C-start, and because of structure-fluid interactions between fish and water, which require the computed fluid forces to be fed back into calculating how the fish moves. Developing robust time-dependent grids and algorithms that do not cause singularities at some point during the motion cycle is a science in itself and very time-consuming.

\section{Conclusions}

So far, very few studies have computed propulsive forces during undulatory swimming for organisms larger than a millimetre: Carling et al. (1998) calculated the two-dimensional flow field around eels, and Liu et al. $(1996,1997)$ calculated the twoand three-dimensional flow field of tadpoles. Although the CFD models predict forces and flow patterns adjacent to the animal that are conceivable, these computed flow fields need to be compared with flow fields recorded around swimming animals if the predicted force patterns are to be believed. However, the wake behind an eel as predicted by Carling et al. (1998) differs substantially from the vortex pattern observed behind swimming eel (Carling et al. 1998; Müller et al. 2001; Tytell 2004a; Tytell and Lauder 2004). The 3-D wake predictions by Liu et al. (1997) for a tadpole have never been tested. Without reliable data on the relationship between body wave and propulsive force generation, the wake hypothesis can only be studied inductively by gathering more data on real fish. We can glean some information from the recorded flow fields alone and from how 
the flow patterns change as the fish swim at different steady swimming speeds and as it changes swimming speed. These hints might help us to answer the question how body wave and body shape affect wake shape or whether all fish converge on the same wake shape because they converged on the optimal Strouhal number for maximum swimming efficiency. To answer the main question about which part of the fish's body acts as propeller and which propulsion mechanism it uses, we are still waiting for reliable force data.

In the meantime, we can explore the existing flow field data to address the optimum wake hypothesis. We have pictures of wakes for a range of undulatory swimmers. These observed wake patterns fall broadly into two categories, one observed mainly in anguilliform (Gray 1933; Hertel 1966; Müller et al. 2001; Tytell 2004a; Tytell and Lauder 2004) and one observed mainly in carangiform swimmers (Blickhan et al. 1992; Müller et al. 1997; Wolfgang et al. 1999; Nauen and Lauder 2002; Wilga and Lauder 2002). Eels generate a wake consisting of two vortex rings per tail beat, irrespective of swimming speed (Tytell 2004a). The increased propulsive force required to swim faster manifests as an increased flow speed through the centre of the vortex ring. The vortex ring itself changes neither its size nor its orientation, ensuring that wake shape remains essentially the same over a fourfold change in swimming speed. Steadily swimming mackerel and mullet generate a chain of vortex rings over a twofold increase in swimming speed (Nauen and Lauder 2002). We conclude that despite similar tail beat kinematics, eels shed a different wake from carangiform swimmers. If we assume that both fish aim at maximizing efficiency during steady swimming, then body shape might play a role in this difference between anguilliform and carangiform wake. In particular, the span of the propeller affects the height of the shed vortex rings and hence the shape of the wake. In eel, the span of the propeller can be approximated by the maximum height of the body. In mullet and mackerel, which generate most of their thrust at the tail, it is the span of the tail fin. If we assume that the height of the vortex ring is proportional to the span of the propeller, then mullet and mackerel generate much taller vortex rings than eel. However, eel, mackerel and mullet all have a similar stride length, tail beat frequency and tail beat amplitude. Therefore, the vortex rings in their wake have a similar width, which can be approximated as the distance between the points at which the tail changes direction and can be computed from the tail beat frequency, tail beat amplitude and swimming speed of the fish (Müller et al. 2001; Nauen and Lauder 2002; Tytell 2004a). When comparing the estimates of vortex ring height with those of ring width, mullet and mackerel shed approximately round vortex rings, whereas eel vortex ring are almost twice as wide as they are tall. Such distorted rings are less likely to form and once formed, they are more likely to break up, all of which results in a different vortex pattern in the wake. The time-resolved flow fields of eels indeed support the notion that the vortices in its wake break up: two independent studies, visualizing horizontal cross-sections through the eel's wake, observed that individual vortex cores elongate and split into two (Müller et al. 2001; Tytell 2004a). We can therefore conclude that the shape of the propeller, and hence implicitly the propulsion mechanism, might affect wake shape. This observation also implies that a propeller shape that promotes the formation of a suboptimal wake is less efficient, and therefore the absence or presence of a wake that is predicted to maximize efficiency is a valuable indicator for the hydrodynamic efficiency of a fish's swimming design.

\section{Conclusions and the next challenges}

\section{How to power undulatory swimming?}

Although we have come a long way towards understanding the mechanics of fish swimming, we still do not have a complete picture of undulatory swimming from muscle contractions to swimming performance. Much progress has been made in recent years quantifying the flow forces acting on a swimming fish, to establish the propulsion mechanisms and to link muscle action to swimming performance. High-speed kinematic analysis and flow visualization have been important stepping stones and CFD models are making considerable progress. As long as in vivo muscle and flow force measurements remain unfeasible, building a forward and inverse dynamic models remain the main options to answer the question how undulatory swimming works (for an overview of forward and inverse dynamic modelling see Otten 2003). The next challenge is to refine existing inverse dynamic models, e.g. by using CFD to obtain flow forces. Several bottlenecks still exist: adult fish swim in a turbulent flow regime, which requires the use of 
turbulence models in the CFD calculations. These difficulties can be avoided when focusing instead on juvenile and larval fish, whose swimming behaviour is unlikely to be fundamentally different from adult fish: all life-history stages face the same hydrodynamic limitations governing the vortex topology of their wake, which in turns determines their swimming performance. Therefore, despite quantitative differences, fish larvae and adult anguilliform fish share a similar locomotory and muscle morphology (van der Meulen et al. 2005), generate similar body waves (Müller and van Leeuwen 2004) and shed similar wakes (Müller et al. 2000). Another challenge is the accurate mapping of the mechanical behaviour of the fish's body. In recent years, there has been considerable progress documenting the body architecture of fish with sufficient detail to build inverse dynamic models that accurately implement the structures of the fish's body. However, the mechanical properties of the various tissues are still largely unknown, and modelling the mechanical behaviour of the complex structures that these tissues form is no trivial matter. Here, the work begun in a.o. Wainwright's research group (e.g. Wainwright et al. 1978) needs to be extended (e.g. Long 1995; Long et al. 1996).

\section{How to control undulatory swimming?}

While we now have a better understanding of how fish generate propulsive forces by using their undulating body and their oscillating tail fin, we still know very little about how undulatory swimming is controlled. Consequently, research into the hydrodynamic mechanisms of swimming is now shifting its focus away from propulsion mechanisms and onto matters of stability and control. Several recent studies have explored how fish control the interaction between the flow patterns generated by their various propulsive surfaces to control their movements, their swimming performance and their stability. Fish generate flow patterns that help them enhance their swimming performance: fish exploit the interaction between the wakes shed by their various fins and trailing edges (e.g. Drucker and Lauder 2001; Müller et al. 2002; Nauen and Lauder 2002). Boxfish and cowfish generate vortices to increase static stability: the keels on their carapace generate corrective flow patterns when the attitude of the fish is disturbed (e.g. Bartol et al. 2005). Fish also exploit the flow generated by other fish or by their environment: Liao et al. (2003a) discovered the so-called Kármán gait, which trout adopt when swimming in the wake of an upstream object. Trout align themselves with the vortices of the oncoming wake and in this manner significantly reduce axial muscle activity (Liao et al. 2003b). In order to exploit the energy of the oncoming vortices, fish need to be able to adapt their motor pattern to create the desired vortex interaction (Liao et al. 2003a).

Fish control their body wave and hence their propulsion by changing the contraction pattern of their swimming muscle. If fish use several propellers at the same time, such as the combination of paired fins and undulatory swimming, then these movements need to be coordinated for the fish to exploit the interaction between the wakes of the these propellers. Hale and her research group (e.g. Thorson et al. 2004) explore the swimming behaviour and neural circuitry of larval fish, which commonly use a combination of pectoral and undulatory swimming during slow bursts. For interactions between self-generated wakes, the fish needs to coordinate the feed-forward central pattern generators that control the swimming movements to achieve the desired performance enhancement from the wake interaction. No sensory feedback is necessary, informing the fish about the selfgenerated flow pattern to affect the desired wake interaction.

However, for the fish to exploit flow patterns in its environment, sensory feedback is mandatory: for a trout to exploit the vortex pattern shed by an upstream obstacle, the fish needs sensory input in order to align itself with the oncoming vortices (Liao 2004). Liao (2004) showed that trout do not simply coast in the lee of obstacles, but that the fish's swimming kinematics matches the obstacle's vortex shedding pattern. EMG studies suggest that this behaviour is not entirely passive (Liao et al. 2003b), hence matching its body movements to the flow pattern requires feedback from the senses that detect the vortex pattern to the motion pattern generators. During the Kármán gait, trout might not use a central pattern generator in the same manner as when swimming normally, making this gait an interesting study object to explore the role of feedback in cyclic locomotion.

\section{How efficient is undulatory swimming?}

Many fish use undulatory swimming for longdistance migration. Under these circumstances, 
propulsive efficiency should become a priority. Evidence for mechanisms that increase hydrodynamic efficiency is slowly being gathered. Fish exploiting the interaction between flows shed of their various body parts, e.g. between the dorsal and tail fin of sunfish (Drucker and Lauder 2001), have been well documented. However, the consequences of such flow manipulations for overall propulsion efficiency remain largely intractable. Studies using mechanical models might allow scientists to measure forces and propulsion efficiency directly. Until then, respiratory studies, which map total locomotory performance and cannot discriminate between the contributions of, e.g. muscle and propeller efficiency, serve to emphasize that there is much to be learnt about how undulatory swimming works (e.g. van Ginneken et al. 2005).

\section{Acknowledgements}

We thank Chuck Hollingworth and John Videler for inviting us to write this review and David Lentink for his comments on the manuscript. UKM was supported by NWO grant (project number 814.02.006).

\section{References}

Akster, H.A. (1985) Morphometry of muscle fibre types in the carp (Cyprinus carpio L.). Relationships between structural and contractile characteristics. Cell and Tissue Research 241, 193-201.

Alexander, R. M. (1969) The orientation of muscle fibres in the myomeres of fishes. Journal of the Marine Biological Association of the United Kingdom 49, 263-290.

Altringham, J.D. and Ellerby, D.J. (1999) Fish swimming: patterns in muscle function. Journal of Experimental Biology 202, 3397-3403.

Bartol, I.K., Gharib, M., Webb, P.W., Weihs, D. and Gordon, M.S. (2005) Body-induced vortical flows: a common mechanism for self-corrective trimming control in boxfishes. Journal of Experimental Biology 208, 327344.

Birch, J.M., Dickson, W.B. and Dickinson, M.H. (2004) Force production and flow structure of the leading edge vortex on flapping wings at high and low Reynolds numbers. Journal of Experimental Biology 207, 10631072.

Blickhan, R., Krick, C., Zehren, D. and Nachtigall, W. (1992) Generation of a vortex chain in the wake of a subundulatory swimming. Naturwissenschaften $\mathbf{7 9}$, 220-221.

Bowtell, G. and Williams, T.L. (1991) Anguilliform body dynamics: modelling the intersection between muscle activation and body curvature. Philosophical Transactions of the Royal Society London B 334, 385-390.

Brainerd, E.L. and Azizi, E. (2005) Muscle fiber angle, segment bulging and architectural gear ratio in segmented musculature. Journal of Experimental Biology 208, 3249-3261.

Carling, J., Williams, T.L. and Bowtell, G. (1998) Selfpropelled anguilliform swimming: simultaneous solution of the two-dimensional Navier-Stokes equations and Newton's laws of motion. Journal of Experimental Biology 201, 3143-3166.

Cheng, J.-Y., Pedley, T.J. and Altringham, J.D. (1998) A continuous dynamic beam model for swimming fish. Philosophical Transactions of the Royal Society London B 353, 981-997.

Coughlin, D.J. (2002) Aerobic muscle function during steady swimming in fish. Fish and Fisheries 3, 63-78.

Coughlin, D.J., Valdez, L. and Rome, L.C. (1996) Muscle length changes during swimming in scup: sonomicrometry verifies the anatomical high-speed cine technique. Journal of Experimental Biology 199, 459-463.

Dewar, H. and Graham, J.B. (1994) Studies of tropical tuna swimming performance in a large water tunnel. III. Kinematics. Journal of Experimental Biology 192, 45-59.

Donley, J.M. and Dickson, K.A. (2000) Swimming kinematics of juvenile kawakawa tuna (Euthynnus affinis) and chub mackerel (Scomber japonicus). Journal of Experimental Biology 203, 3103-3116.

Donley, J.M., Sepulveda, C.A., Konstantinidis, P., Gemballa, S. and Shadwick, R.E. (2004) Convergent evolution in mechanical design of lamnid sharks and tunas. Nature 429, 61-65.

Drucker, E.G. and Jensen, J.S. (1996) Pectoral fin locomotion in striped surfperch. II. Scaling swimming kinematics and performance at gait transition. Journal of Experimental Biology 199, 2243-2252.

Drucker, E.G. and Lauder, G.V. (1999) Locomotor forces on swimming fish: three-dimensional vortex wake dynamics quantified using digital particle velocimetry. Journal of Experimental Biology 202, 2393-2412.

Drucker, E.G. and Lauder, G.V. (2001) Locomotor function of the dorsal fin in teleost fishes: experimental analysis of wake forces in sunfish. Journal of Experimental Biology 204, 2943-2958.

Ellerby, D.J. and Altringham, J.D. (2001) Spatial variation in fast muscle function of the rainbow trout Oncorhynchus mykiss during start-starts and sprinting. Journal of Experimental Biology 204, 2239-2250.

Fierstine, H.L. and Walters, V. (1968) Studies in the locomotion and anatomy of scombrid fishes. Memoirs of the Southern California Academy of Sciences 6, 131.

Fuiman, L.A. and Batty, R.S. (1997) What a drag it is getting cold: partitioning the physical and physiological effects of temperature on fish swimming. Journal of Experimental Biology 200, 1745-1755. 
Gemballa, S. and Röder, K. (2004) From head to tail: the myoseptal system in basal actinopterygians. Journal of Morphology 259, 155-171.

van Ginneken, V., Antonissen, E., Müller, U.K., Booms, R., Eding, E., Verreth, J. and van den Thillart, G. (2005) Eel migration to the Sargasso: remarkably high swimming efficiency and low energy costs. Journal of Experimental Biology 208, 1329-1335.

Goldbogen, J.A., Shadwick, R.E., Fudge, D.S. and Gosline, J.M. (2005) Fast-start muscle dynamics in the rainbow trout Oncorhynchus mykiss: phase relationship of white muscle shortening and body curvature. Journal of Experimental Biology 208, 929-938.

Gopalkrishnan, R., Triantafyllou, M.S., Triantafyllou, G.S. and Barrett, D. (1994) Active vorticity control in a shear flow using a flapping foil. Journal of Fluid Mechanics 174, $1-21$.

Graham, J.B., Koehrn, F.J. and Dickson, K.A. (1983) Distribution and relative proportions of red muscle in scombrid fishes: consequences of body size and relationships to locomotion and endothermy. Canadian Journal of Zoology 61, 2087-2096.

Gray, J. (1933) Studies in animal locomotion. I. The movement of the fish with special reference to the eel. Journal of Experimental Biology 10, 88-103.

Greene, C.W. and Greene, C.H. (1913) The skeletal musculature of king salmon. Bulletin of the United States Fish Commission 33, 21-59.

Hertel, H. (1966) Structure, Form and Movement. Reinhold Publishing Corp., New York.

Hess, F. and Videler, J.J. (1984) Fast continuous swimming of saithe (Pollachius virens): a dynamic analysis of bending moments and muscle power. Journal of Experimental Biology 109, 229-251.

Johnston, I.A. (1991) Muscle action during locomotion: a comparative perspective. Journal of Experimental Biology 160, $167-185$.

Johnston, I.A., Davison, W. and Goldspink, G. (1977) Energy-metabolism of carp swimming muscles. Journal of Comparative Physiology 114, 203-216.

Jordan, C.E. (1992) A model of rapid-start swimming at intermediate Reynolds numbers: undulatory swimming in the chaetognath Sagitta elegans. Journal of Experimental Biology 163, 119-137.

Jordan, C.E. (1996) Coupling internal and external mechanics to predict swimming behaviour: a general approach? American Zoologist 36, 710-722.

Josephson, R.K. (1985) The mechanical power output from striated muscle during cyclic contraction. Journal of Experimental Biology 114, 493-512.

Katz, S.L., Shadwick, R.E. and Rapoport, H.S. (1999) Muscle strain histories in swimming milkfish in steady and sprinting gaits. Journal of Experimental Biology 202, 529-541.

Lamb, H. (1932) Hydrodynamics. Cambridge University Press, Cambridge.
Lauder, G.V. and Drucker, E.G. (2002) Forces, fishes and fluids: hydrodynamic mechanisms of aquatic locomotion. News in Physiological Sciences 17, 235-240.

van Leeuwen, J.L. (1995) The action of muscles in swimming fish. Review article. Experimental Physiology 80, 177-191.

van Leeuwen, J.L. (1999) A mechanical analysis of myomere shape in fish. Journal of Experimental Biology 202, 3405-3414.

van Leeuwen, J.L. and Spoor, C.W. (1992) Modelling mechanically stable muscle architectures. Philosophical Transactions of the Royal Society London B 336, 275 292.

van Leeuwen, J.L. and Spoor, C.W. (1993) Modelling the pressure and force equilibrium in unipennate muscles with in-line tendons. Philosophical Transactions of the Royal Society London B 342, 321-333.

van Leeuwen, J.L., Lankheet, M.J.M., Akster, H.A. and Osse, J.W.M. (1990) Function of red axial muscles of carp (Cyprinus carpio): recruitment and normalized power output during swimming in different modes. Journal of Zoology London 220, 123-145.

Liao, J.C. (2004) Neuromuscular control of trout swimming in a vortex street: implications for energy economy during the Kármán gait. Journal of Experimental Biology 207, 3495-3506.

Liao, J.C., Beal, D.N., Lauder, G.V. and Triantafyllou, M.S. (2003a) The Kármán gait; novel kinematics of rainbow trout swimming in a vortex street. Journal of Experimental Biology 206, 1059-1073.

Liao, J.C., Beal, D.N., Lauder, G.V. and Triantafyllou, M.S. (2003b) Fish exploiting vortices decrease muscle activity. Science 302, 1566-1569.

Lighthill, M.J. (1960) Note on the swimming of slender fish. Journal of Fluid Mechanics 9, 305-317.

Lighthill, M.J. (1969) Hydrodynamics of aquatic animal propulsion - a survey. Annual Review of Fluid Mechanics 1, 413-446.

Lindsey, C.C. (1978) Form, function, and locomotory habits in fish. In: Fish Physiology, Vol. VII (eds W.S. Hoar and D.J. Randall). Academic Press, New York, pp. 1100.

Liu, H., Wassersug, R.J. and Kawachi, K. (1996) A computational fluid dynamics study of tadpole swimming. Journal of Experimental Biology 199, 1245-1260.

Liu, H., Wassersug, R.J. and Kawachi, K. (1997) The three-dimensional hydrodynamics of tadpole locomotion. Journal of Experimental Biology 200, 2807-2819.

Long, J.H. (1995) Morphology, mechanics, and locomotion: the relation between the notochord and swimming speed in sturgeon. Environmental Biology of Fishes 44, 199-211.

Long, J.H., Hale, M.E., McHenry, M.J. and Westneat, M.W. (1996) Functions of fish skin: flexural stiffness and steady swimming of longnose gar Lepisosteus osseus. Journal of Experimental Biology 199, 2139-2151. 
Long, J.H., Adcock, B. and Root, R.G. (2002a) Force transmission via axial tendons in undulating fish: a dynamic analysis. Comparative Biochemistry and Physiology A 133, 911-929.

Long, J.H., Roots, R.G. and Watts, P. (2002b) Is an undulating fish an oscillating wing? Integrative and Comparative Biology 42, 1268.

Malkiel, E., Sheng, I., Katz, J. and Strickler, J.R. (2003) The three-dimensional flow field generated by a feeding calanoid copepod measured using digital holography. Journal of Experimental Biology 206, 3657-3666.

McHenry, M.J. and Lauder, G.V. (2005) Mechanical scaling of coasting in zebrafish (Danio rerio). Journal of Experimental Biology 208, 2289-2301.

McHenry, M.J., Azizi, E. and Strother, J.A. (2003) The hydrodynamics of locomotion at intermediate Reynolds numbers: undulatory swimming in ascidian larvae (Botrylloides sp.). Journal of Experimental Biology 206, 327-343.

van der Meulen, T., Schipper, H., van Leeuwen, J.L. and Kranenbarg, S. (2005) Effects of decreased muscle activity on developing axial musculature in nic $^{\text {b107 }}$ mutant zebrafish (Danio rerio). Journal of Experimental Biology 208, 3675-3687.

Müller, U.K. and van Leeuwen, J.L. (2004) Swimming of larval zebrafish: ontogeny of body waves and implications for locomotory development. Journal of Experimental Biology 207, 853-868.

Müller, U.K., den Heuvel, B.L.E., Stamhuis, E.J. and Videler, J.J. (1997) Fish footprints: morphology and energetics of the wake behind a continuously swimming mullet (Chelon labrosus Risso). Journal of Experimental Biology 200, 2893-2906.

Müller, U.K., Stamhuis, E.J. and Videler, J.J. (2000) Hydrodynamics of unsteady fish swimming and the effects of body size: comparing fish larvae and adults. Journal of Experimental Biology 203, 193-206.

Müller, U.K., Smit, J., Stamhuis, E.J. and Videler, J.J. (2001) How the body contributes to the wake in undulatory fish swimming: flow fields of a swimming eel (Anguilla anguilla). Journal of Experimental Biology 204, 2751-2762.

Müller, U.K., Stamhuis, E.J. and Videler, J.J. (2002) Riding the waves: the role of the body wave in undulatory fish swimming. Integrative and Comparative Biology 42, 981987.

Nauen, J.C. and Lauder, G.V. (2001) Locomotion in scombrid fishes: visualisation of flow around the caudal peduncle and finlets of the chub mackerel Scomber japonicus. Journal of Experimental Biology 204, 22512263.

Nauen, J.C. and Lauder, G.V. (2002) Hydrodynamics of caudal fin locomotion by chub mackerel, Scomber japonicus (Scombridae). Journal of Experimental Biology 205, 1709-1724.

Noca, F., Shiels, D. and Jeon, D. (1997) Measuring instantaneous fluid dynamic forces on bodies, using only velocity fields and their derivatives. Journal of Fluids and Structures 11, 345-350.

Otten, E. (2003) Inverse and forward dynamics: models of multi-body systems. Philosophical Transactions of the Royal Society London B 358, 1493-1500.

Pedley, T.J. and Hill, S.J. (1999) Large-amplitude undulatory fish swimming: fluid mechanics coupled to internal mechanics. Journal of Experimental Biology 202, 34313438.

Pereira, F., Gharib, M., Dabiri, D. and Modarress, D. (2000) Defocusing digital particle image velocimetry: a 3component 3-dimensional DPIV measurement technique. Application to bubbly flows. Experiments in Fluids 29, S78-S84.

Prandtl, L. and Tietjens, O.G. (1934) Fundaments of Hydroand Aerodynamics. Dover Publications, New York.

Rome, L.C. and Sosnicki, A.A. (1991) Myofilament overlap in swimming carp. II. Sarcomere length changes during swimming. American Journal of Physiology 260, C289C296.

Rome, L. C., Swank, D. and Corda, D. (1993) How fish power swimming. Science 261, 340-343.

Schultz, W.W. and Webb, P.W. (2002) Power requirements of swimming: do new methods resolve old questions? Integrative and Comparative Biology $\mathbf{4 2}$, 1018-1025.

Schultz, W.W., Zhou, Q.-N. and Webb, P.W. (1991) A twodimensional model of fish swimming. Journal of the Marine Biological Association 71, 739.

Shadwick, R.E., Rapoport, H.S. and Fenger, J.M. (2002) Structure and function of tuna tail tendons. Comparative Biochemistry and Physiology A 133, 1109-1125.

Spedding, G. R., Rayner, J.M.V. and Pennycuick, C.J. (1984) Momentum and energy in the wake of a pigeon (Columbia livia) in slow flight. Journal of Experimental Biology 111, 81-102.

van der Stelt, A. (1968) Spiermechanica en myotoombouw bij vissen. PhD thesis. L. J. Veen's Uitgeversmaatschappij N.V., University of Amsterdam, Amsterdam. [in Dutch]. Streitlien, K., Triantafyllou, G.S. and Triantafyllou, M.S. (1996) Efficient foil propulsion through vortex control. AIAA Journal 34, 2315-2318.

Thorson, D.H., Cassidy, J.J., Hale, M.E. et al. (2004) Swimming of larval zebrafish: fin-axis coordination and implications for function and neural control. Journal of Experimental Biology 207, 4175-4183.

Triantafyllou, M.S., Triantafyllou, G.S. and Gopalkrishnan, R. (1991) Wake mechanics for thrust generation in oscillation foils. Physics of Fluids A 3, 2835-2837.

Triantafyllou, G.S., Triantafyllou, M.S. and Grosenbaugh, M.A. (1993) Optimal thrust development in oscillating foils with application to fish propulsion. Journal of Fluids and Structures 7, 205-224.

Tytell, E.D. (2004a) The hydrodynamics of eel swimming. II. Effect of swimming speed. Journal of Experimental Biology 207, 3265-3279. 
Tytell, E.D. (2004b) Kinematics and hydrodynamics of linear acceleration in eels, Anguilla rostrata. Philosophical Transactions of the Royal Society London B 271, 25352540.

Tytell, E.D. and Lauder, G.V. (2004) Hydrodynamics of eel swimming. I. Wake structure. Journal of Experimental Biology 207, 1825-1841.

Videler, J.J. (1993) Fish swimming. Chapman and Hall, London.

Videler, J.J. and Hess, F. (1984) Fast continuous swimming of two pelagic predators, saithe (Pollachius virens) and mackerel (Scomber scombrus): a kinematic analysis. Journal of Experimental Biology 109, 209-228.

Wainwright, S.A. (1983) To bend a fish. In: Fish Biomechanics (eds P.W. Webb and D. Weihs). Praeger Publishers, New York, pp. 68-91.

Wainwright, S.A., Vosburgh, F. and Hebrank, J.H. (1978) Shark skin: function in locomotion. Science 202, 747749.

Wakeling, J.M. and Johnston, I.A. (1999) White muscle strain in the common carp and red to white muscle gearing ratios in fish. Journal of Experimental Biology 202, 521-528.
Wardle, C.S., Videler, J.J. and Altringham, J.D. (1995) Tuning in to fish swimming waves: body form, swimming mode and muscle function. Journal of Experimental Biology 198, 1629-1636.

Westneat, M.W., Hoese, W., Pell, C.A. and Wainwright, S.A. (1993) The horizontal septum: mechanisms of force transfer in locomotion of scombrid fishes (Scombridae, Peciformes). Journal of Morphology 217, 183-204.

Wilga, C.D. and Lauder, G.V. (2002) Function of the heterocercal tail in sharks: quantitative wake dynamics during steady horizontal swimming and vertical maneuvering. Journal of Experimental Biology 205, 2365-2374.

Wolfgang, M.J., Anderson, J.M., Grosenbaugh, M.A., Yue, D.K.P. and Triantafyllou, M.S. (1999) Near-body flow dynamics in swimming fish. Journal of Experimental Biology 202, 2303-2327.

Wu, T.Y. (1961) Swimming of a waving plate. Journal of Fluid Mechanics 10, 321-344.

Yates, G.T. (1983) Hydromechanics of body and caudal fin propulsion. In: Fish Biomechanics (eds P.W. Webb and D. Weihs). Praeger Publishers, New York, pp. 177-213. 